\section{Der Beitrag von Komorbidität und sozialer Belastung zur Notwendig- keit einer Krankenhausaufnahme wegen Störungen durch psychotrope Substanzen psychotrope Substanzen}

Zusammenfassung: Bei Krankenhausaufnahme wegen Störungen durch psychotrope Substanzen wurden in 22 psychiatrischen Institutionen an $\mathbf{n}=699$ Patienten Entzugssymptomatik, psychische, neurologische und weitere somatische Komorbidität sowie soziale Belastungen getrennt erhoben. Für jeden dieser Bereiche wurde das Ausmaß der aktuellen Beeinträchtigung und einer sich daraus ergebenden Notwendigkeit von Krankenhausbehandlung eingeschätzt.

Eine eindeutige Aufnahmeindikation ergab sich aufgrund des Entzugs in 59,9\%, einer weiteren psychischen Störung in $53,1 \%$, einer weiteren neurologischen Symptomatik in $\mathbf{8 , 7} \%$ und aufgrund einer anderweitigen somatischen Erkrankung in 24,5\%. Aktueller Hilfebedarf bezüglich sozialer und rechtlicher Probleme bestand in 38,8\% $(n=271)$ der Aufnahmen. Bei Opiatkonsumenten ( $n=183$ ) trugen die neurologische und die psychiatrische Komorbidität weniger und der Entzug sowie die weitere somatische Komorbidität mehr zur Notwendigkeit von Krankenhausbehandlung bei als bei Alkoholkonsumenten $(n=497)$.

Schlüsselwörter: Komorbidität - Krankenhausaufnahme Psychotrope Substanzen - Alkohol - Sucht

Social Impairment, Somatic, and Psychiatric Co-morbidity in Substance Abuse- Related Intakes: To which amount do social handicaps and different fields of co-morbidity contribute to the necessity of in-patient treatment in substance abusers?

In 22 German psychiatric departments 699 of 750 sequential substance abuse related intakes (19.9\% females) were assessed by a structured interview and clinical examination. The average age was 38.3 . $96 \%$ met ICD-10 criteria of dependency. There was a need of in-patient treatment because of withdrawal in $59.9 \%$, psychiatric disorders in $53.1 \%$, neurological symptoms in $8.7 \%$, and further medical signs in $24.5 \% .38 .8 \%$ needed professional help because of social impairment. Friedman' $s$ test confirmed the largest impact for withdrawal (rank (r): 3.86) and for psychiatric co-morbidity (r: 3.49), followed by actual need for help concerning social impairment (r: 3.16).

Compared to alcohol related intakes confined to legal drugs $(n=497)$, in heroine addicts $(n=183)$ neurological and psychiatric co-morbidity contributed to a smaller, but withdrawal, further medical symptoms, and social impairment to a

Suchttherapie 2000; 1: 98-103

(c) Georg Thieme Verlag Stuttgart · New York ISSN 1439-9903
Gerhard Reymann ${ }^{1}$, Helga Spranger, Robert Stracke², Andrea Harnisch ${ }^{3}$, Kersten Schulz ${ }^{4}$, Kristina Pulkkinen ${ }^{5}$, Marion Brand ${ }^{1}$, Volkmar Goldammer ${ }^{6}$, Wilhelm Unkel ${ }^{7}$, Michael Müller-Mohnssen ${ }^{8}$

Aus der Suchtmedizinischen oder der Psychiatrischen Abteilung der/des

1 WZPPP Dortmund, ${ }^{2}$ Klinikum Nord Hamburg,

${ }^{3}$ Städt. Krhs. Eisenhüttenstadt, ${ }^{4}$ Fachklinik Schleswig,

${ }^{5}$ Psychiatr. Krhs. Rickling, ${ }^{6}$ Landesklinik Brandenburg,

${ }^{7}$ Landeskrankenhaus Wunstorf, ${ }^{8}$ ZfP Weissenau

larger amount to in-patient treatment necessity (MannWhitney Test: $\mathrm{p}<0.001$ SPSS 8 ).

Key words: Co-Morbidity - In-Patient Treatment - Psychotropic Substances - Alcohol - Addiction

\section{Einleitung}

Das Ausmaß sozialer Belastungen und die Bedeutung von somatischer und psychiatrischer Komorbidität bei Konsumenten psychotroper Substanzen ist hinreichend bekannt [1-4]. Wenig untersucht ist dagegen, mit welchen Problemen und entsprechend auch mit welchen Zielsetzungen die Betroffenen vorrangig Krankenhausbehandlung in Anspruch nehmen.

In dieser Studie wird untersucht, in welchem Ausmaß die verschiedenen Komponenten von Komorbidität und auch soziale Suchtfolgeschäden zur Notwendigkeit von Krankenhausbehandlung beitragen.

\section{Material und Methode}

Die Erhebung wurde an 23 Institutionen der stationären psychiatrischen Pflichtversorgung aus 8 Bundesländern (BRB, BW, HH, NRW, NS, SCN, SH, SLD) durchgeführt. Dabei handelt es sich um 16 Fachkrankenhäuser, 7 psychiatrische Fachabteilungen und eine Universitätsklinik.

Die Fachkrankenhäuser und die Universitätsklinik wiesen spezielle Stationen zur Behandlung Abhängigkeitskranker auf. Eingeschlossen wurden alle während des Untersuchungszeitraumes in diese Stationen aufgenommenen Patienten. Sofern in den psychiatrischen Abteilungen keine für die Behandlung Abhängigkeitskranker spezialisierten Stationen abgegrenzt waren, wurden diejenigen Patienten eingeschlossen, bei denen die substanzbedingte Störung bzw. eine typische Suchtfolgeerkrankung wesentlicher Aufnahmegrund war.

Die Erhebung wurde fortlaufend über einen Zeitraum von 14 Tagen durchgeführt. Innerhalb dieses Zeitraumes wiederholt aufgenommene Personen wurden nur bei der ersten Aufnahme erfasst. Der Institution stand es frei, den Untersuchungszeitraum zwischen dem 1.10.1999 und dem 30.11.1999 prospektiv selbst zu bestimmen. Eine Einrichtung legte den Untersuchungszeitraum auf einen späteren Zeitpunkt. 
Die Erfassungsquote bleibt aufgrund der unscharfen Definition der in den nicht suchtmedizinisch spezialisierten Stationen einzuschließenden Patienten ein Schätzwert. Eine Einrichtung (psychiatrisches Fachkrankenhaus) wurde aufgrund einer Erfassungsrate unter 60\% nicht in die Auswertung einbezogen. In den verbleibenden 22 Einrichtungen konnten von ca. 750 Aufnahmen 699 (ca. 93\%) Datensätze erhoben werden. 139 (19,9\%) der Untersuchten waren weiblich, $560(80,1 \%)$ männlich. Das mittlere Alter betrug 38,3 Jahre (Frauen 38,6, Männer 38,2) mit einer Standardabweichung von 11,7 .

Die Daten wurden mit einem Fragebogen erhoben, der von den behandelnden Ärzten in der Regel am ersten Werktag nach der stationären Aufnahme ausgefüllt wurde. Es wurde somit der Kenntnisstand abgefragt, der zum Zeitpunkt der Erstellung des Behandlungsplanes tatsächlich vorhanden war. Er umfasste die jeweils verfügbaren eigen- und fremdanamnestischen Angaben, den klinischen Befund und die Verlaufsbeobachtung der Anfangsphase der Behandlung. Laborbefunde und Ergebnisse technischer Untersuchungen wurden einbezogen, sofern sie notfallmäßig angefertigt oder vorbekannt waren.

Der Fragebogen bezog sich zunächst auf die Abhängigkeitsdiagnose nach ICD 10 (6 Items) [5], das Konsumverhalten (32 Items) und die Behandlungsvorerfahrung (7 Items). In den Abschnitten Entzugssymptomatik (5 Items), weitere psychische Symptomatik (19 Items), weitere neurologische Symptomatik (8 Items), weitere somatische Erkrankungen (20 Items), soziale und rechtliche Belastungen (29 Items) [6] sowie Motivation (3 Items) und Ressourcen wurden zusammenfassend jeweils Einschätzungen auf einer Skala zwischen 0 und 9 vorgenommen. Die statistische Auswertung erfolgte mit SPSS 8.

\section{Ergebnisse}

\section{Diagnose des Abhängigkeitssyndroms}

Bei 28 Aufnahmen (4,0\%) lag keine stoffgebundene Abhängigkeit vor. Auch diese Patienten wurden in die weiteren Berechnungen mit einbezogen. 543 Patienten (77,7\%) erfüllten mehr als die für die Diagnosestellung erforderlichen drei Kriterien und bei 278 Patienten (39,8\%) trafen alle sechs ICD-10-Kriterien $\mathrm{zu}$.

\section{Konsumverhalten}

Bei der Abfrage wurde zwischen Haupt- und Nebensubstanzen unterschieden. Als Hauptsubstanz wurde das Suchtmittel oder die Stoffgruppe aufgefasst, das oder die wesentlich zur Krankenhausaufnahme beigetragen hatte. Die Beantwortung der Frage nach den einzelnen Kriterien der Abhängigkeit gemäß ICD-10 bezog sich auf die Hauptsubstanz.

Bei 562 Aufnahmen (80,4\%) legten sich die aufnehmenden Ärzte auf eine Hauptsubstanz oder eine Substanzgruppe fest. Hierbei handelte es sich in 445 (63,7\% von 699 eingeschlossenen Patienten) Fällen um Alkohol, in 99 Fällen (14,2\%) um Opiate, in 6 Fällen $(0,9 \%)$ um Medikamente mit Suchtpotenzial (ausschließlich Opiatsubstitution) und in 12 Fällen $(1,7 \%)$ um andere Suchtmittel.
In 101 Fällen (14,5\%) wurden zwei Hauptsubstanzen genannt. Bei 31 (4,4\%) der Aufnahmen standen drei und bei $5(0,7 \%)$ alle vier der genannten Substanz(-gruppen) gleichwertig nebeneinander.

Bei Nennung von Alkohol als Hauptsubstanz betrug die Trinkmenge durchschnittlich $252 \mathrm{~g}$ pro Tag.

Als Nebensubstanz wurde ein Suchtmittel eingestuft, das nicht wesentlich zur Indikation für eine Krankenhausaufnahme beitrug. So wurde zum Beispiel Tabak nur einmal als Haupt-, aber 445-mal als Nebensubstanz eingeschätzt.

\section{Ausmaß der Störungen und Belastungen}

Tab. 1 zeigt für jede der fünf geprüften Dimensionen die Verteilung der Scorewerte, ihren Median, ihren Mittelwert und ihren nach dem Friedman-Test für K-verbundene Stichproben ermittelten Rangwert. Das Entzugssyndrom, in das ein etwaiges Delir und Entzugskrämpfe mit einbezogen wurden, stellte gemeinsam mit den weiteren psychischen Störungen den Hauptanteil der aktuellen Belastungen dar.

$71,7 \%$ der Untersuchten wiesen keine weitere neurologische Symptomatik auf. Bei $64,2 \%$ war zumindest eine leichte Störung aus einem der weiteren somatischen Fachgebiete evident. Die Bedeutung der sozialen und der rechtlichen Probleme wurde von den Ärzten höher als die der weiteren somatischen Erkrankungen, aber geringer als die des Entzuges und die der psychischen Komorbidität eingeschätzt.

Der Anteil der Patienten ohne abstinente Sozialkontakte war bei den vorwiegend Alkohol konsumierenden Patienten $(\mathrm{n}=497)$ und den Opiatabhängigen $(n=183)$ ähnlich $(29,2 \%$ versus $31,5 \%$ ). Beide Gruppen unterschieden sich aber signifikant zum Beispiel bezüglich des Bezuges von Sozialhilfe (25,5\% versus $39,5 \%$ ) oder bezüglich illegaler Einkünfte einschließlich Prostitution (5,8\% versus 60,6).

\section{Die Indikation zur Krankenhausbehandlung}

Die Notwendigkeit von Krankenhausbehandlung wies besonders bei entzugsbezogenen, bei psychiatrischen und bei neurologischen Störungen eine hohe Korrelation mit der Schwere der entsprechenden Symptomatik auf (siehe Tab. 2). Bei den weiteren somatischen Störungen und besonders in Bezug auf soziale Belastungen war diese Korrelation geringer.

Die fünf Dimensionen korrelierten untereinander mit Koeffizienten stets kleiner als 0,3 und zum Teil nur auf einem Signifikanzniveau von $\mathrm{p}>0,01$.

Patienten, denen mehrere Hauptsubstanzen zugeordnet waren (siehe Tab.3), zeigten im Durchschnitt keinen höheren stationären Behandlungsbedarf bezüglich des Entzugssyndroms als solche mit nur einer. Erstere hatten in $80 \%$ bereits früher einen stationären Entzug in Anspruch genommen, Letztere in 62\%. Möglicherweise kamen Patienten, bei denen im Rahmen von Vorbehandlungen ein adäquates Verhalten bei Rückfällen thematisiert worden war [7], vermehrt zur Aufnahme, bevor sich wesentliche Entzugserscheinungen und -komplikationen ausgebildet hatten. 
Tab.1 Die Schwere der aktuellen Beeinträchtigung durch die Entzugssymptomatik, komorbide Störungen und soziale Belastungen eingeschätzt nach einem Score von 0 bis 9 mit dafür berechnetem Median, Mittelwert und mittlerem Rangwert (bestimmt nach dem Friedman-Test für K-verbundene Stichproben)

\begin{tabular}{|c|c|c|c|c|c|c|c|c|c|c|c|c|c|}
\hline & \multicolumn{2}{|c|}{ nicht vorhanden } & \multicolumn{2}{|c|}{$\begin{array}{l}\text { leicht } \\
\text { ausgeprägt }\end{array}$} & \multicolumn{2}{|c|}{$\begin{array}{l}\text { mittlere } \\
\text { Ausprägung }\end{array}$} & \multicolumn{2}{|c|}{$\begin{array}{l}\text { starke } \\
\text { Ausprägung }\end{array}$} & \multicolumn{2}{|c|}{$\begin{array}{l}\text { extreme } \\
\text { Ausprägung }\end{array}$} & \multirow[t]{2}{*}{ Median } & \multirow{2}{*}{$\begin{array}{l}\text { Mittel- } \\
\text { Wert } \\
\text { (Stabw.) }\end{array}$} & \multirow{2}{*}{$\begin{array}{l}\text { mittlerer } \\
\text { Rang- } \\
\text { wert }\end{array}$} \\
\hline & Score 0 & Score 1 & Score 2 & Score 3 & Score 4 & Score 5 & Score 6 & Score 7 & Score 8 & Score 9 & & & \\
\hline $\begin{array}{l}\text { Ausprägung der } \\
\text { Entzugs- } \\
\text { symptomatik }\end{array}$ & $\begin{array}{l}18,6 \% \\
(n=130)\end{array}$ & $\begin{array}{l}5,0 \% \\
(n=35)\end{array}$ & $\begin{array}{c}9,4 \% \\
(n=66)\end{array}$ & $\begin{array}{l}10,3 \% \\
(n=72)\end{array}$ & $\begin{array}{l}10,9 \% \\
(n=76)\end{array}$ & $\begin{array}{l}12,0 \% \\
(n=84)\end{array}$ & $\begin{array}{l}13,9 \% \\
(n=97)\end{array}$ & $\begin{array}{l}10,4 \% \\
(n=73)\end{array}$ & $\begin{array}{l}5,9 \% \\
(n=41)\end{array}$ & $\begin{array}{l}3,6 \% \\
(n=25)\end{array}$ & 4 & $\begin{array}{l}3,94 \\
(2,71)\end{array}$ & 3,69 \\
\hline $\begin{array}{l}\text { Ausprägung } \\
\text { weiterer psychi- } \\
\text { scher Störungen }\end{array}$ & $\begin{array}{l}21,7 \% \\
(n=152)\end{array}$ & $\begin{array}{l}3,6 \% \\
(n=25)\end{array}$ & $\begin{array}{c}6,7 \% \\
(n=47)\end{array}$ & $\begin{array}{l}11,9 \% \\
(n=83)\end{array}$ & $\begin{array}{l}16,3 \% \\
(n=114)\end{array}$ & $\begin{array}{l}13,6 \% \\
(n=95)\end{array}$ & $\begin{array}{l}10,6 \% \\
(n=74)\end{array}$ & $\begin{array}{c}9,4 \% \\
(n=66)\end{array}$ & $\begin{array}{l}3,4 \% \\
(n=24)\end{array}$ & $\begin{array}{l}2,7 \% \\
(n=19)\end{array}$ & 4 & $\begin{array}{l}3,68 \\
(2,59)\end{array}$ & 3,46 \\
\hline $\begin{array}{l}\text { Ausprägung } \\
\text { weiterer neuro- } \\
\text { logischer } \\
\text { Störungen }\end{array}$ & $\begin{array}{l}71,7 \% \\
(n=501)\end{array}$ & $\begin{array}{l}4,9 \% \\
(n=34)\end{array}$ & $\begin{array}{c}5,9 \% \\
(n=41)\end{array}$ & $\begin{array}{c}6,9 \% \\
(n=48)\end{array}$ & $\begin{array}{c}4,3 \% \\
(n=30)\end{array}$ & $\begin{array}{c}2,3 \% \\
(n=16)\end{array}$ & $\begin{array}{c}2,0 \% \\
(n=14)\end{array}$ & $\begin{array}{r}0,6 \% \\
(n=4)\end{array}$ & $\begin{array}{l}0,7 \% \\
(n=5)\end{array}$ & $\begin{array}{l}0,9 \% \\
(n=6)\end{array}$ & 0 & $\begin{array}{l}0,95 \\
(1,85)\end{array}$ & 1,86 \\
\hline $\begin{array}{l}\text { Ausprägung } \\
\text { weiterer } \\
\text { somatischer } \\
\text { Störungen }\end{array}$ & $\begin{array}{l}35,8 \% \\
(n=250)\end{array}$ & $\begin{array}{l}6,2 \% \\
(n=43)\end{array}$ & $\begin{array}{l}12,3 \% \\
(n=86)\end{array}$ & $\begin{array}{l}13,3 \% \\
(n=93)\end{array}$ & $\begin{array}{l}11,7 \% \\
(n=82)\end{array}$ & $\begin{array}{c}6,9 \% \\
(n=48)\end{array}$ & $\begin{array}{c}6,6 \% \\
(n=46)\end{array}$ & $\begin{array}{c}4,1 \% \\
(n=29)\end{array}$ & $\begin{array}{l}1,6 \% \\
(n=11)\end{array}$ & $\begin{array}{l}1,6 \% \\
(n=11)\end{array}$ & 2 & $\begin{array}{l}2,47 \\
(2,43)\end{array}$ & 2,77 \\
\hline $\begin{array}{l}\text { Ausprägung } \\
\text { sozialer und } \\
\text { rechtlicher } \\
\text { Probleme }\end{array}$ & $\begin{array}{l}22,5 \% \\
(n=157)\end{array}$ & $\begin{array}{l}6,4 \% \\
(n=45)\end{array}$ & $\begin{array}{l}12,2 \% \\
(n=85)\end{array}$ & $\begin{array}{l}13,3 \% \\
(n=93)\end{array}$ & $\begin{array}{l}13,4 \% \\
(n=94)\end{array}$ & $\begin{array}{c}8,7 \% \\
(n=61)\end{array}$ & $\begin{array}{l}10,4 \% \\
(n=73)\end{array}$ & $\begin{array}{c}8,0 \% \\
(n=56)\end{array}$ & $\begin{array}{l}3,2 \% \\
(n=22)\end{array}$ & $\begin{array}{l}1,9 \% \\
(n=13)\end{array}$ & 3 & $\begin{array}{l}3,29 \\
(2,55)\end{array}$ & 3,23 \\
\hline
\end{tabular}

Tab. 2 Die Indikation zur Krankenhausaufnahme aufgrund der Entzugssymptomatik, der komorbiden Störungen und des aktuellen sozialen Hilfebedarfes eingeschätzt nach einem Score von 0 bis 9 mit dafür berechnetem Median, Mittelwert und mittlerem Rangwert (bestimmt nach dem Friedman-Test für K-verbundene Stichproben) sowie die Korrelation mit der Ausprägung der Beeinträchtigung auf dem entsprechenden Feld gemäß Tab. 1

\begin{tabular}{|c|c|c|c|c|c|c|c|c|c|c|c|}
\hline \multicolumn{2}{|c|}{$\begin{array}{l}\text { keine } \\
\text { Indikation }\end{array}$} & $\begin{array}{l}\text { fragliche } \\
\text { Khs.-Indikation }\end{array}$ & \multicolumn{2}{|c|}{$\begin{array}{l}\text { Khs.- Behandlung } \\
\text { empfohlen }\end{array}$} & \multirow{2}{*}{$\begin{array}{l}\text { Khs.- Behandlung } \\
\text { notwendig } \\
\text { Score } 6 \text { Score } 7\end{array}$} & \multicolumn{2}{|c|}{$\begin{array}{l}\text { absolute } \\
\text { Indikation }\end{array}$} & \multirow[b]{2}{*}{ Median } & \multirow[b]{2}{*}{$\begin{array}{l}\text { Mittel- } \\
\text { Wert } \\
\text { (Stabw.) }\end{array}$} & \multirow[b]{2}{*}{$\begin{array}{l}\text { mitt- } \\
\text { lerer } \\
\text { Rang- } \\
\text { wert }\end{array}$} & \multirow{2}{*}{$\begin{array}{l}\text { Korrelationzur } \\
\text { Ausprägung } \\
\text { der Störung } \\
\text { Korrelations- } \\
\text { koeffizient } \\
(p<0,001)\end{array}$} \\
\hline Score 0 & Score 1 & Score 2 Score 3 & Score 4 & Score 5 & & Score 8 & Score 9 & & & & \\
\hline \multicolumn{12}{|c|}{ stationäre Behandlungsbedürftigkeit der Entzugssymptomatik } \\
\hline $\begin{array}{l}19,5 \% \\
(n=136)\end{array}$ & $\begin{array}{l}4,6 \% \\
(n=32)\end{array}$ & $\begin{array}{cc}7,0 \% & 9,0 \% \\
(n=49) & (n=63)\end{array}$ & $\begin{array}{l}10,3 \% \\
(n=72)\end{array}$ & $\begin{array}{l}11,7 \% \\
(n=82)\end{array}$ & $\begin{array}{ll}12,5 \% & 12,7 \% \\
(n=87) & (n=89)\end{array}$ & $\begin{array}{l}7,0 \% \\
(n=49)\end{array}$ & $\begin{array}{l}5,7 \% \\
(n=40)\end{array}$ & 4 & $\begin{array}{l}4,17 \\
(2,86)\end{array}$ & 3,86 & 0,862 \\
\hline \multicolumn{12}{|c|}{ stationäre Behandlungsbedürftigkeit weiterer psychischer Störungen } \\
\hline $\begin{array}{l}24,6 \% \\
(n=172)\end{array}$ & $\begin{array}{l}4,0 \% \\
(n=28)\end{array}$ & $\begin{array}{cc}8,6 \% & 9,7 \% \\
(n=60) & (n=68)\end{array}$ & $\begin{array}{l}14,5 \\
(n=101)\end{array}$ & $\begin{array}{l}12,6 \% \\
(n=88)\end{array}$ & $\begin{array}{ll}10,2 \% & 9,3 \% \\
(n=71) & (n=65)\end{array}$ & $\begin{array}{l}2,9 \% \\
(n=20)\end{array}$ & $\begin{array}{l}3,7 \% \\
(n=26)\end{array}$ & 4 & $\begin{array}{l}3,54 \\
(2,70)\end{array}$ & 3,49 & 0,824 \\
\hline \multicolumn{12}{|c|}{ stationäre Behandlungsbedürftigkeit weiterer neurologischer Störungen } \\
\hline $\begin{array}{l}75,8 \% \\
(n=530)\end{array}$ & $\begin{array}{l}4,1 \% \\
(n=29)\end{array}$ & $\begin{array}{cc}5,6 \% & 5,7 \% \\
(n=39) & (n=40)\end{array}$ & $\begin{array}{c}3,2 \% \\
(n=22)\end{array}$ & $\begin{array}{c}1,9 \% \\
(n=13)\end{array}$ & $\begin{array}{cl}1,7 \% & 0,7 \% \\
(n=12) & (n=5)\end{array}$ & $\begin{array}{l}0,4 \% \\
(n=3)\end{array}$ & $\begin{array}{l}0,9 \% \\
(n=6)\end{array}$ & 0 & $\begin{array}{l}0,81 \\
(1,74)\end{array}$ & 1,93 & 0,879 \\
\hline \multicolumn{12}{|c|}{ stationäre Behandlungsbedürftigkeit weiterer somatischer Störungen } \\
\hline $\begin{array}{l}46,9 \% \\
(n=328)\end{array}$ & $\begin{array}{l}8,3 \% \\
(n=58)\end{array}$ & $\begin{array}{lc}11,6 \% & 8,7 \% \\
(n=81) & (n=61)\end{array}$ & $\begin{array}{c}8,4 \% \\
(n=59)\end{array}$ & $\begin{array}{c}4,6 \% \\
(n=32)\end{array}$ & $\begin{array}{cl}5,2 \% & 3,6 \% \\
(n=36) & (n=25)\end{array}$ & $\begin{array}{l}1,1 \% \\
(n=8)\end{array}$ & $\begin{array}{l}1,6 \% \\
(n=11)\end{array}$ & 1 & $\begin{array}{l}1,94 \\
(2,39)\end{array}$ & 2,56 & 0,790 \\
\hline \multicolumn{12}{|c|}{ stationäre Behandlungsbedürftigkeit sozialer und rechtlicher Probleme } \\
\hline $\begin{array}{l}30,8 \% \\
(n=215)\end{array}$ & $\begin{array}{l}8,2 \% \\
(n=57)\end{array}$ & $\begin{array}{ll}9,7 \% & 12,6 \% \\
(n=68) & (n=88)\end{array}$ & $\begin{array}{l}10,2 \% \\
(n=71)\end{array}$ & $\begin{array}{c}7,9 \% \\
(n=55)\end{array}$ & $\begin{array}{cl}8,6 \% & 6,2 \% \\
(n=60) & (n=43)\end{array}$ & $\begin{array}{l}3,2 \% \\
(n=22)\end{array}$ & $\begin{array}{l}2,9 \% \\
(n=20)\end{array}$ & 3 & $\begin{array}{l}2,91 \\
(2,68)\end{array}$ & 3,16 & 0,671 \\
\hline
\end{tabular}


Tab. 3 Der Score zur Einschätzung der Notwendigkeit einer Krankenhausbehandlung aufgrund des/der konsumierten Suchtmittel/-s differenziert nach Entzugssymptomatik, komorbiden Störungen und aktuellem sozialen Hilfebedarf $(n=699)$

\begin{tabular}{|c|c|c|c|c|c|c|c|c|c|c|c|}
\hline & & \multicolumn{2}{|c|}{ Entzugssymptomatik } & \multicolumn{2}{|c|}{$\begin{array}{l}\text { weitere psychische } \\
\text { Störungen }\end{array}$} & \multicolumn{2}{|c|}{$\begin{array}{l}\text { weitere neurologische } \\
\text { Störungen }\end{array}$} & \multicolumn{2}{|c|}{$\begin{array}{l}\text { weitere somatische } \\
\text { Störungen }\end{array}$} & \multicolumn{2}{|c|}{$\begin{array}{l}\text { sozialer Hilfe- } \\
\text { bedarf }\end{array}$} \\
\hline & & Mittelw. & Stabw. & Mittelw. & Stabw. & Mittelw. & Stabw. & Mittelw. & Stabw. & Mittelw. & Stabw. \\
\hline $\begin{array}{l}\text { Hauptsubstanz } \\
\text { Alkohol }\end{array}$ & $\mathrm{n}=445$ & 4,02 & 3,00 & 3,56 & 2,69 & 1,04 & 1,98 & 1,82 & 2,33 & 2,59 & 2,61 \\
\hline $\begin{array}{l}\text { Hauptsubstanz } \\
\text { Opiate }\end{array}$ & $n=99$ & 5,01 & 2,12 & 2,60 & 2,62 & 0,25 & 0,82 & 1,91 & 2,47 & 3,34 & 2,80 \\
\hline $\begin{array}{l}\text { Hauptsubstanz } \\
\text { Medikamente }\end{array}$ & $\mathrm{n}=6$ & & & & & & & & & & \\
\hline $\begin{array}{l}\text { Hauptsubstanz } \\
\text { weitere Suchtmittel }\end{array}$ & $\mathrm{n}=12$ & & & & & & & & & & \\
\hline $\begin{array}{l}\text { mehrere } \\
\text { Hauptsubstanzen } \\
\text { ohne Opiate }\end{array}$ & $n=53$ & 3,38 & 2,67 & 4,47 & 2,55 & 1,00 & 1,71 & 2,08 & 2,26 & 3,26 & 2,65 \\
\hline $\begin{array}{l}\text { mehrere } \\
\text { Hauptsubstanzen } \\
\text { inkl. Opiate }\end{array}$ & $\mathrm{n}=84$ & 4,77 & 2,70 & 3,68 & 2,65 & 0,29 & 0,87 & 2,55 & 2,53 & 3,71 & 2,60 \\
\hline Summe & $n=699$ & 4,17 & 2,86 & 3,54 & 2,70 & 0,81 & 1,74 & 1,94 & 2,39 & 2,91 & 2,68 \\
\hline
\end{tabular}

In allen anderen Kategorien boten die Patienten, die mehrere Hauptsubstanzen parallel konsumierten, gleichen oder höheren Behandlungsbedarf.

Bei Opiatkonsumenten haben der Entzug, die sozialen Belastungen und die somatische Komorbidität eine größere Bedeutung als bei Patienten, die Alkohol, aber keine Opiate konsumieren (siehe Tab.4). Dagegen hat bei Alkoholkonsumenten die psychiatrische und auch die neurologische Komorbidität größere Bedeutung für die Notwendigkeit der Krankenhausbehandlung. Diese Ergebnisse sind nach dem nichtparametrischen Mann-Whitney-U-Test mit $\mathrm{p}<0,001$ signifikant.

\section{Diskussion}

Bei der Aufnahme in psychiatrische Krankenhausbehandlung aufgrund von Störungen durch psychotrope Substanzen stellt die Entzugssymptomatik die schwer wiegendste Beeinträchtigung dar. Die Tatsache, dass sie bei Alkoholkonsumenten weniger stark als bei Opiatabhängigen ausgeprägt ist, kann damit zusammenhängen, dass Erstere ganz überwiegend intoxikiert zur Aufnahme kommen und die Entwicklung von Entzugskomplikationen durch eine rechtzeitige adäquate Medikation vermindert wird, während sich Letztere nicht selten aufgrund der schlechteren Verfügbarkeit der Opiate erst viele Stunden nach dem letzten Konsum zur Aufnahme vorstellen. Sofern - wie bei 83,6\% der hier untersuchten Opiatabhängigen - keine ambulante Methadon- oder Polamidonsubstitution besteht, hat sich zum Aufnahmezeitpunkt oft bereits ein deutliches Entzugssyndrom entwickelt.

Ebenfalls hoch wurde die Bedeutung der weiteren psychiatrischen Komorbidität eingeschätzt. Bei $52,4 \%$ ergab sie eine eindeutige Behandlungsindikation.

Wienberg et al. [8] fanden 1991 im Verlauf von 977 psychiatrischen Krankenhausbehandlungen Abhängigkeitskranker in 67,9\% mindestens eine komorbide psychiatrische Erkrankung. Berücksichtigt man, dass in diesem Anteil das Delirium tremens (5,3\% der Patienten) und das schwere Entzugssyn-

Tab. 4 Gegenüberstellung des Scores der Indikation zur Krankenhausbehandlung aufgrund von vier Bereichen der Komorbidität und des sozialen Hilfebedarfes für opiatfreie Alkoholkonsumenten $(n=497)$ und Opiatkonsumenten $(n=183)$. Statistische Berechnung nach SPSS 8 mit dem MannWhitney-U-Test

\begin{tabular}{|c|c|c|c|c|c|c|}
\hline & \multicolumn{3}{|c|}{ Alkoholkonsumenten ohne Opiatkonsum ( $n=497$ ) } & \multicolumn{2}{|c|}{ Opiatkonsumenten $(n=183)$} & \multirow[b]{2}{*}{ Rangsumme } \\
\hline & Score (Stabw.) & mittlerer Rang & Rangsumme & Score (Stabw.) & mittlerer Rang & \\
\hline $\begin{array}{l}\text { Entzugs } \\
\text { Symptomatik }\end{array}$ & $\begin{array}{l}3,96 \\
(2,97)\end{array}$ & 324,0 & 161025,0 & $\begin{array}{l}4,90 \\
(2,40)\end{array}$ & 385,3 & 70515,0 \\
\hline weitere psychische Störung & $\begin{array}{l}3,66 \\
(2,69)\end{array}$ & 358,1 & 177974,0 & $\begin{array}{l}3,09 \\
(2,68)\end{array}$ & 292,7 & 53566,0 \\
\hline weitere neurologische Störung & $\begin{array}{l}1,03 \\
(1,95)\end{array}$ & 351,3 & 174633,5 & $\begin{array}{l}0,27 \\
(0,84)\end{array}$ & 311,0 & 56906,5 \\
\hline weitere somatische Störung & $\begin{array}{l}1,84 \\
(2,33)\end{array}$ & 334,0 & 165974,5 & $\begin{array}{l}2,20 \\
(2,51)\end{array}$ & 358,3 & 65565,5 \\
\hline $\begin{array}{l}\text { sozialer } \\
\text { Hilfebedarf }\end{array}$ & $\begin{array}{l}2,66 \\
(2,62)\end{array}$ & 323,9 & 160980,0 & $\begin{array}{l}3,51 \\
(2,71)\end{array}$ & 385,6 & 70560,0 \\
\hline
\end{tabular}


drom gemäß ICD-9 (21,9\%) mit enthalten sind, da damals für Entzugskomplikationen keine gesonderte Rubrik geführt wurde, ist eher eine Zunahme der psychiatrischen Komorbidität wahrscheinlich. Dies ist auch deshalb anzunehmen, da die damalige Untersuchung sämtliche Befunde einer im Durchschnitt 29,6 Tage umfassenden stationären Behandlung nutzte.

Felduntersuchungen lassen ebenfalls hohe Raten der psychiatrischen Komorbidität bei Krankenhausaufnahme erwarten. So belief sich die auf die Lebenszeit bezogene Komorbidität von Alkoholabhängigkeit für mindestens eine weitere psychiatrische Erkrankung bei einer standardisierten Befragung von 8098 zufällig ausgewählten Personen im Alter zwischen 15 und 54 Jahren bei Frauen auf 86,0\% (Odds Ratio 7,4) und bei Männern auf 78,3\% (Odds Ratio 5,6) [4]. Diese Ergebnisse sind mit der hier gefundenen Punktprävalenz vereinbar, wenn Krankenhausaufnahme bevorzugt beim Auftreten oder einer Exazerbation einer komorbiden Störung in Anspruch genommen wird.

In der Versorgungsforschung wird Komorbidität bevorzugt an Kollektiven untersucht, die sich in längerfristiger Behandlung befinden. Krausz et al. [3] fanden bei 350 Opiatabhängigen, die ganz überwiegend in langfristige Behandlungskonzepte der Hamburger Drogenhilfe integriert waren, eine 2-WochenPrävalenz für Störungen nach ICD-10 F2X bis F5X von 23\%.

Die hier vorgestellte Untersuchung hat ein weit geringeres Maß an diagnostischer Sicherheit, da die Datenerhebung zu einem Zeitpunkt erfolgt, der durch Intoxikation sowie Entzug belastet ist und der kaum den Einsatz umfangreicherer standardisierter Messinstrumente erlaubt. Dennoch sind die Ergebnisse gut miteinander vereinbar, wenn, wie oben angenommen wird, bei einer akuten Symptomatik im Rahmen einer komorbiden psychiatrischen Störung Krankenhausbehandlung vermehrt in Anspruch genommen wird und wenn man berücksichtigt, dass bei der Hamburger Untersuchung die bei Opiatkonsumenten zahlenmäßig besonders bedeutsamen Persönlichkeitsstörungen [9] nicht erfasst wurden.

Arnold et al. [10] fanden bei 2258 Aufnahmen von Patienten mit Drogenproblemen in somatischen Krankenhausabteilungen lediglich in 5,3\% eine psychiatrische Diagnose. Ob Suchtmittelkonsumenten, die somatische Krankenhäuser aufsuchen, tatsächlich sehr viel seltener psychische Störungen aufweisen oder ob psychiatrische Störungen dort $\mathrm{zu}$ wenig beachtet werden, bleibt offen.

Die neurologische Komorbididät ist bei Konsum von Alkohol aufgrund seiner großen Neurotoxizität [11] stärker als bei Opiatabhängigen. Sie trägt insgesamt wenig zur Aufnahmeindikation bei, da die bei 138 Patienten relevanten Krampfanfälle $\mathrm{zu} 76,7 \%$ als Entzugssymptom erfasst wurden. Auch ist die Polyneuropathie - das mit 139 Nennungen (19,8\% der Patienten) in dieser Studie häufigste neurologische Krankheitsbild - nur bei schwerer Ausprägung Indikation für eine Krankenhausbehandlung. In der Studie von Wienberg et al. [8] war trotz eines höheren Anteiles Alkoholabhängiger diese Störung nur bei 13,5\% der Patienten gesehen worden.
Arnold et al. [10] beschreiben für ihre Stichprobe aus Allgemeinkrankenhäusern, dass somatische Diagnosen in mehr als 55\% die Krankenhausaufnahme rechtfertigten. Die Differenz zu den $24,5 \%$, die in der hier vorgelegten Untersuchung aufgrund von weiteren somatischen Erkrankungen der Krankenhausbehandlung bedurften, beruht auf mehreren Faktoren: Zum einen wurde in der Untersuchung von Arnold et al. die Notwendigkeit der Krankenhausbehandlung aus den Entlassungsdiagnosen erschlossen, während in dieser Studie nur die zum Aufnahmezeitpunkt verfügbaren Daten berücksichtigt wurden. So war hier zum Beispiel lediglich bei 39,5\% der Patienten mit Alkoholproblemen ein Leberschaden evident. Arnold et al. fanden in ihrer Stichprobe trotz einer geringeren durchschnittlichen Trinkmenge aufgrund pathologischer Laborwerte in 78,2\% einen Leberschaden. Zum anderen werden Suchtmittelkonsumenten, die zum Beispiel der Unterstützung der Vitalfunktionen eines chirurgischen Eingriffes oder der gezielten Behandlung von Infektionskrankheiten [12] bedürfen, fast ausschließlich in den dafür spezialisierten Fachabteilungen behandelt.

Der auf Sozialhilfe angewiesene Anteil bei den Patienten mit vorrangigen Alkoholproblemen, aber ohne Opiatkonsum, lag bei Aufnahme in somatische Krankenhausbehandlung bei 15,0\% [10], bei Aufnahme in die bei dieser Studie kooperierenden psychiatrischen Krankenhäuser und Fachabteilungen bei $24,1 \%$. Die entsprechenden Werte für Opiatkonsumenten betrugen 56,6\% und 60,6\%. Abstinente Sozialkontakte fehlten bei Alkoholkranken in rund $10 \%$ in der somatischen und rund $28 \%$ in der psychiatrischen Krankenhausbehandlung.

Die Ergebnisse dieser Untersuchung sprechen dafür, dass in der Psychiatrie bei Aufnahmen wegen Störungen durch psychotrope Substanzen zusätzlich die Behandlung des Entzugssyndroms und der psychiatrischen Komorbidität im Vordergrund stehen. Das Klientel ist stärker von sozialen Suchtfolgeschäden belastet als in somatischen Abteilungen. Da somatische Folgeerkrankungen bei Aufnahme oft nicht evident sind, ist eine ausreichende Diagnostik im Behandlungsverlauf notwendig.

\section{Danksagung}

Unser Dank gilt: Herrn B. Bätz, Warstein; Herrn Joachim Tegeler, Leipzig; Herrn H. Elsner, Gelsenkirchen; Herrn F. Andritsch, Weinsberg; Herrn P. Schwalvenberg, Hemer; Herrn J. P. Marcea, Zülpich; Herrn G. Kruse, Hannover; Herrn Thomas Birker, Heide; Herrn Dirk Schwoon, Hamburg; Herrn K. Behrendt, Hamburg; Herrn S. Rinkens, Frankfurt/O.; Herrn Christoph Göhlert, Neuruppin; Herrn Wolfgang Werner, Merzig; Frau Gudrun Richter, Angermünde und Herrn Peter Rochler, Görlitz

\section{Literatur}

${ }^{1}$ Prinzleve M. Problembelastung und Hilfebedarf von obdachlosen Drogenabhängigen aus der offenen Hamburger Drogenscene. Hamburg: NOX Jugendhilfe e.V. Kirchenalle 25, 20099 Hamburg, 2000

2 Murray CJL, Lopez AD. Global mortality, disability, and the contribution of risk factors: Global Burden of Disease Study. The Lancet 1997; 349: 1436-1442 
${ }^{3}$ Krausz M, Verthein U, Degkwitz P. Prävalenz psychischer Störungen bei Opiatabhängigen mit Kontakt zum Drogenhilfesystem. Nervenarzt 2000; 69: 557-567

${ }^{4}$ Kessler RC, Crum RM, Warner LA, Nelson CB, Schulenberg J, Anthony JC. Lifetime co-occurrence of DSM-III-R alcohol abuse and dependence with other psychiatric disorders in the National Comorbidity Survey. Arch Gen Psychiatry 1997; 54: 313-321

${ }^{5}$ WHO ICD 10 Internationale statistische Klassifikation der Krankheiten und verwandter Gesundheitsprobleme. Amtliche deutschsprachige Ausgabe 10. Revision. Deutsches Institut für Medizinische Dokumentation und Information. Köln, Bern: Hans Huber, 1999

${ }^{6}$ Arbeitsgruppe CMA. Definitionsvorschlag zur Operationalisierung von chronisch mehrfachbeeinträchtigten Abhängigen von psychotropen Substanzen. Sucht 1999; 45: 6-13

7 Körkel J. Umgang mit dem Rückfall. In: Gölz J. Moderne Suchtmedizin (Hrsg). Stuttgart, New York: Thieme, 1999

${ }^{8}$ Wienberg G, Andritsch F, Bertram W, Drees E, Fleischmann H, Gerber HG, von der Haar M, Kruse G, Pörksen N, Schlösser A, Spranger H, Steinberg R, Zechert C. Abhängigkeitskranke in psychiatrischer Krankenhausbehandlung - Ergebnisse einer Erhebung in 14 Kliniken der Bundesrepublik. Sucht 1993; 4: 264-275

${ }^{9}$ Krausz M. Komorbidität von psychischen Störungen und Sucht. In: Gölz J (Hrsg). Der drogenabhängige Patient, München, Jena: Urban und Fischer, 1999

${ }^{10}$ Arnold T, Schmid M, Simmedinger R. Suchthilfe im Krankenhaus - Endbericht der wissenschaftlichen Begleitung des Bundesmodellprogramms „Drogennotfallprophylaxe/Nachgehende Sozialarbeit“. Baden- Baden: Nomos Verlagsgesellschaft, 1999

${ }^{11}$ Seitz H, Lieber C, Simanowski U. Handbuch Alkohol, Alkoholismus, Alkoholbedingte Organschäden. Leipzig, Heidelberg: Johann A Barth, 1995

12 Baumgarten R. Stationäre Betreuung suchtkranker Patienten. In: Gölz J (Hrsg). Der drogenabhängige Patient, München, Jena: Urban und Fischer, 1999
Dr. med. Gerhard Reymann

WZPPP Dortmund

Marsbruchstraße 179

44287 Dortmund

E-mail: gerhard.reymann@wkp-lwl.org 